\title{
Diseño de un Sistema Experto Difuso para la Determinación de la Densidad de Corriente en una Planta de Cromado
}

\author{
Carolina V. Ponce y Bayron Rojas \\ Fac. De Ingeniería, Dpto. de Ingeniería Mecánica, Universidad De La Serena, Benavente 980, La Serena- \\ Chile. (e-mail: cponce@userena.cl; bayron_17@live.cl)
}

Recibido Jul. 25, 2018; Aceptado Oct. 10, 2018; Versión final Nov. 12, 2018, Publicado Abr. 2019

\begin{abstract}
Resumen
Se diseñó un sistema experto difuso, para determinar la densidad de corriente y su tiempo de aplicación en una planta de cromado duro. Primeramente, se realiza un análisis de la teoría general de cromado duro y se contrasta con la ejecución práctica del mismo. Se escogen las variables relevantes del proceso, las que luego son modeladas mediante lógica difusa, definiendo funciones de pertenencia, rangos específicos y una base de reglas que describe el sistema. Se utiliza un sistema del tipo Mamdani para la modelación de la densidad de corriente y su tiempo de aplicación del proceso de cromado duro. El sistema experto obtenido mostró un porcentaje de error promedio, entre el proceso real y el modelo, no superior al $10 \%$. Se concluye que el modelo propuesto es representativo del sistema actual de cromado estudiado. Además, su implementación significaría una disminución de costos asociados, tanto en el ahorro en horas extras del operario de la planta, como de la eficiencia en el uso de la energía.
\end{abstract}

\section{Design of a Fuzzy Expert System for Determination of the Current Density in a Chromium Plant}

\begin{abstract}
A fuzzy expert system was designed for modeling the current density in a hard chrome plating plant. First, an analysis of the general theory of hard chrome is carried out and it is compared with the practical execution of the plating process. The most relevant process variables are selected. After that, a model using fuzzy logic is proposed. To do this, membership functions, specific ranges, and a rule base describing the system, are defined. A Mamdani type system is used for modeling current density and its application time in the hard chrome process. The expert system obtained show an average percent error between the actual process and the model calculations lower than $10 \%$. It is concluded that the proposed model is representative of the current chromed system studied. In addition, its implementation would mean a decrease in costs associated with both the saving of overtime of the plant operator as well as with the efficiency in the use of energy.
\end{abstract}

Keywords: fuzzy logic; expert systems, hard chrome; process modeling 


\section{INTRODUCCIÓN}

Un sistema experto se puede definir como aquel programa de computación, ya sea un software o hardware, que contiene el conocimiento de un especialista humano acerca de un determinado campo de aplicación. Esto es, un modelo computarizado de las capacidades de razonamiento y habilidades en resolución de problemas del especialista humano (Llata et al., 2000). Un sistema experto basado en reglas es aquel que modela la base de conocimiento en un conjunto de reglas que se extraen como resultado de una serie de pruebas experimentales del tema estudiado. En cuanto a la base de reglas, éstas constituyen la más sencilla de las metodologías utilizadas en sistemas expertos. La base de conocimiento contiene el conjunto de reglas que definen el problema, y el motor de inferencia saca las conclusiones aplicando la lógica clásica a estas reglas. Las ventajas que otorga el uso de este tipo de sistemas es que son sencillos de programar una vez que se conoce el conjunto de reglas, y que sus respuestas son extremadamente rápidas.

En cuanto a las desventajas, los sistemas expertos basados en reglas exigen un elevado esfuerzo humano para su puesta en marcha, y que una variación de las funciones de densidad y la inclusión de nuevos elementos, precisa de un nuevo estudio y replanteamiento de las reglas (Pino et al., 2001). Para evitar el problema de reprogramar las reglas ante un cambio del planteamiento del problema, Vaschetti et al., (2012) utilizan un lenguaje basado en reglas denominado CLIPS (C language Integrated Production System) desarrollado por la Nasa. Se presenta el desarrollo de un sistema experto difuso, que reemplaza al experto humano en una etapa del proceso de cromado duro de una planta de cromado. Específicamente, el sistema experto calcula la densidad de corriente y su tiempo de aplicación en el proceso de cromado, operación que actualmente la realiza un operador humano que calcula estas variables sólo de acuerdo a su experiencia. El proceso de cromado duro consiste en aplicar directamente en la parte metálica una capa dura de cromo, resistente al desgaste, con un espesor del orden de micrómetros, sin que sea necesario aplicar capas de cobre o níquel. El cromado posee altos niveles de dureza y resistencia al desgaste, por lo que se puede utilizar en diversos equipos. Este proceso requiere muchos pasos preliminares, tales como: la elección del electrolito y la ubicación, forma y número de electrodos. Estos parámetros afectan el espesor del depósito y la distribución de placas. Los pasos preliminares para optimizar un proceso de recubrimiento pueden consumir mucho tiempo si se realizan con prueba y error (Druesne, 2003). Esto ha llevado al diseño de tecnologías que permiten analizar el proceso de cromado, o francamente buscar nuevas técnicas de cromado que reemplacen el proceso de cromado duro por otro más económico o menos contaminante.

Zhu et al., (2009) y Qian et al., (2010) proponen un método de micro mecanización electroquímica de micro orificios o matriz de hoyuelos, en el que una placa de aislamiento estampada recubierta con una película de cromo duro se une estrechamente a la placa de la pieza de trabajo. La tecnología propuesta ofrece ventajas como plazo de ejecución corto y bajo costo. El efecto de los parámetros del proceso en la forma de la microestructura fue demostrado numéricamente y experimentalmente. Para verificar el efecto de la matriz de micro-hoyuelos en el cromo duro, se realizaron pruebas de fricción utilizando un ensayador de fricción. Aydin et al (2014), realizaron un estudio con el objetivo de investigar la potencialidad del proceso de cromado con cepillo, de forma de reemplazar el cromado duro convencional como una alternativa más ecológica, ya que este último es un proceso bastante contaminante. Lyphout et al., (2014) realizaron una serie de experimentos utilizando la tecnología de rociado térmico como reemplazo del método electrolítico de cromo duro. La menor energía térmica y mayor energía cinética de las partículas pulverizadas logradas con el sistema desarrollado de combustible supersónico de aire, denominado HVAF-M3, reduce significativamente la descarburación y aumenta el desgaste y las propiedades de resistencia a la corrosión, lo que hace que los recubrimientos rociados con HVAF sean atractivos tanto económica y ambientalmente. Establecen relaciones entre configuraciones de hardware, procesos y variables de ingeniería, y propiedades de los recubrimientos. Se enfatiza la relevancia de estos factores del proceso y su importancia. Se discute la optimización de los recubrimientos para mejorar el desgaste por abrasión y el rendimiento de la corrosión. Vinokurov et al., (2016) realizan un análisis de la eficiencia y competitividad de dos tecnologías electroquímicas de cromado. Determinan las fortalezas y debilidades de cada tecnología utilizando el análisis FODA y definen indicadores de calidad de la eficiencia de cada tecnología.

Por otro lado, varios autores han realizado modelos del proceso de cromado o de parte de éste. Pam et al., (1981) realizan el modelado de un electrolito y describen la medición de los sobrepotenciales en soluciones de cromo hexavalente y realizan el análisis de la información resultante. Ruiz et al., (2003) presenta un modelo estadístico que describe el comportamiento del espesor del recubrimiento de cromo duro de acero inoxidable martensítico como función del tiempo de exposición, la densidad de corriente y la temperatura; aplicado a un equipo quirúrgico. El modelo se ajusta por medio de un diseño experimental rotacional compuesto de segundo orden y tiene un poder de explicación del $75 \%$ en la variabilidad de la respuesta. Sánchez et al., (2010) construyen un modelo predictivo del espesor de la capa de cromo en un proceso de cromado duro. Para ello utiliza un modelo de redes neuronales, el cual mejora a través de la técnica de diseño de experimentos (DOE. Design Of Experiments). Obaid et al., (2013) realizan la modelación de un proceso de cromado duro aplicado 
a componentes de automóviles. Para realizar la modelación utilizaron COMSOL Multiphysics y MATLAB. La simulación estudia el efecto de la conductividad de la solución, el espaciamiento de los electrodos y la altura del ánodo, utilizando un enfoque de diseño factorial. Realizan un análisis de sensibilidad para estudiar el efecto de estas variables en el espesor de la superficie de cromo. Concluyen que estas tres variables seleccionadas tienen un gran impacto en el espesor y la uniformidad del cromado. Análogamente, Sánchez et al., (2014) también implementa un modelo predictivo del espesor de la capa de cromo en un proceso de cromado duro. Para ello utiliza un algoritmo de regresión vectorial de soporte evolutivo (SVR: Support Vector Machines) y compara los resultados con resultados anteriores dados en Sánchez et al., (2010). La resistencia a la corrosión de una placa de cromo duro depende del grosor de su recubrimiento, su adherencia y las microfisuras. Esta estructura micro-fisurada es lo que proporciona la dureza óptima de las capas. Kullawong y Butdee (2015), aplicaron la metodología de costo de mantenimiento centrado en la confiabilidad (RCM .Reliability-Centered Maintenance) para la optimización del mantenimiento y gestión de costos para una planta de cromo duro. El objetivo es administrar los costos necesarios para extender la vida útil de una planta a través del uso de métodos probabilísticos y técnicas de simulación, para identificar mejor la importancia de cada componente de la planta con respecto a los costos de mantenimiento. Como resultado de esta investigación, el modelo de costos permite desarrollar una metodología para determinar los costos de mantenimiento que deben aplicarse a algunos subconjuntos de los elementos de una planta.

Otros autores se han enfocado en la automatización de los procesos de cromado. Telukdaire, (2007), desarrolla una herramienta híbrida matemática-difusa para la evaluación de producción limpia en el acabado de superficies, como el cromado duro. Este estudio se centra en proporcionar un sistema de auditoría alternativo fácil de usar para la implementación de la producción más limpia en la industria del acabado metálico. El sistema de auditoría propuesto elimina la necesidad de contar con un auditor técnico y con datos de planta rígidos. El sistema propuesto funciona únicamente en las entradas del operador de la planta. El conocimiento del operador se aprovecha y se utiliza para llevar a cabo una auditoría de producción más limpia, eficiente y efectiva. La investigación se basa en el conocimiento experto, que se obtuvo mediante la realización de auditorías en unas 25 empresas que utilizan herramientas de auditoría tradicionales. Vargas, (2010) desarrolla un sistema de visión artificial para controlar la calidad de piezas cromadas. El sistema se basa en el sistema LabWIEW, el cual mediante una tarjeta de adquisición de datos, realiza la comunicación entre la etapa de percepción y actuación del sistema. El sistema desarrollado permite una 100\% de inspección de piezas, además de disminuir los errores humanos y los costos asociados a un producto en malas condiciones. Mayacela y Otañez, (2013), diseñan un sistema de pulido electrolítico de materiales ferrosos para un proceso de cromado. Para este proceso se controlan la densidad de corriente, temperatura del baño y tiempo de permanencia.

La modelación y simulación de algunos o todos los pasos de un proceso de cromado duro, permite no solo reducir costos, sino también su automatización, lo que conlleva a una optimización del proceso de cromado, lo que significa una mayor eficiencia energética y mejores condiciones de trabajo para el operador, considerando que el proceso de cromado es contaminante. Este trabajo presenta el diseño de un sistema experto difuso para una planta de cromado duro. Utilizando el conocimiento del experto humano del proceso de cromado duro, se desarrolla por medio de la identificación difusa un modelo para calcular la densidad de corriente necesaria para realizar el cromado duro y el tiempo de aplicación de esta corriente. A través de este modelo, se crea un sistema experto que reemplaza al experto humano en el proceso de cálculo y aplicación de la densidad de corriente de la pieza a cromar. Esto no solo le permite al experto humano estar menos tiempo en contacto con los contaminantes que produce el proceso de cromado, sino también optimizar los tiempos de cromado disminuyendo así los costos del proceso.

\section{MATERIALES Y MÉTODOS}

En general los estudios realizados con respecto a los procesos de cromado se refieren a la determinación del espesor de la capa de cromado duro (Sánchez, 2014; Obaid et al., 2013, Sánchez, 2010; Ruíz et al., 2003). A diferencia de estos autores, este trabajo se enfoca en la determinación automática de la densidad de corriente y el tiempo de aplicación de ésta, de forma de hacer más eficiente el proceso de cromado completo.

\section{Descripción de la planta de cromado}

El proceso de cromado se realiza en una tina construida de polipropileno de 10 [mm] de espesor, Debido a sus dimensiones, la tina está instalada en un pozo de concreto de superficie cuadrada que contiene a su vez una tina de acero con dimensiones similares donde va la tina de cromado y cuyo espacio intermedio es utilizado para el control de temperatura a través de un baño maría. La celda de cromado está compuesta por ocho ánodos hechos de una aleación de plomo y estaño. Los ánodos cuelgan con un gancho de cobre conectado a un bus de barras de cobre que cierran el circuito conectándose a un transformador - reductor. La solución electrolítica empleada para el proceso de cromado duro está compuesta por: Ácido Crómico 
(HEFF 25 RS y HEFF 25 AS), Fumetrol, Ácido Sulfúrico, Metasulfito Sódico, Catalizador (HEFF 25C). Se emplea un sistema de control de emisiones a partir de esferas de polipropileno, vertidas en la superficie de la solución electrolítica. Este sistema impide la formación de burbujas de gran dimensión y su aparente explosión, disminuyendo consigo la cantidad de emisiones de ácido crómico liberado a la atmosfera. La figura 1 muestra un esquema del proceso de cromado que va desde el calentamiento de la solución electrolítica hasta el desmontaje de la pieza. Se incluye en la figura las variables que forman parte del modelo difuso.

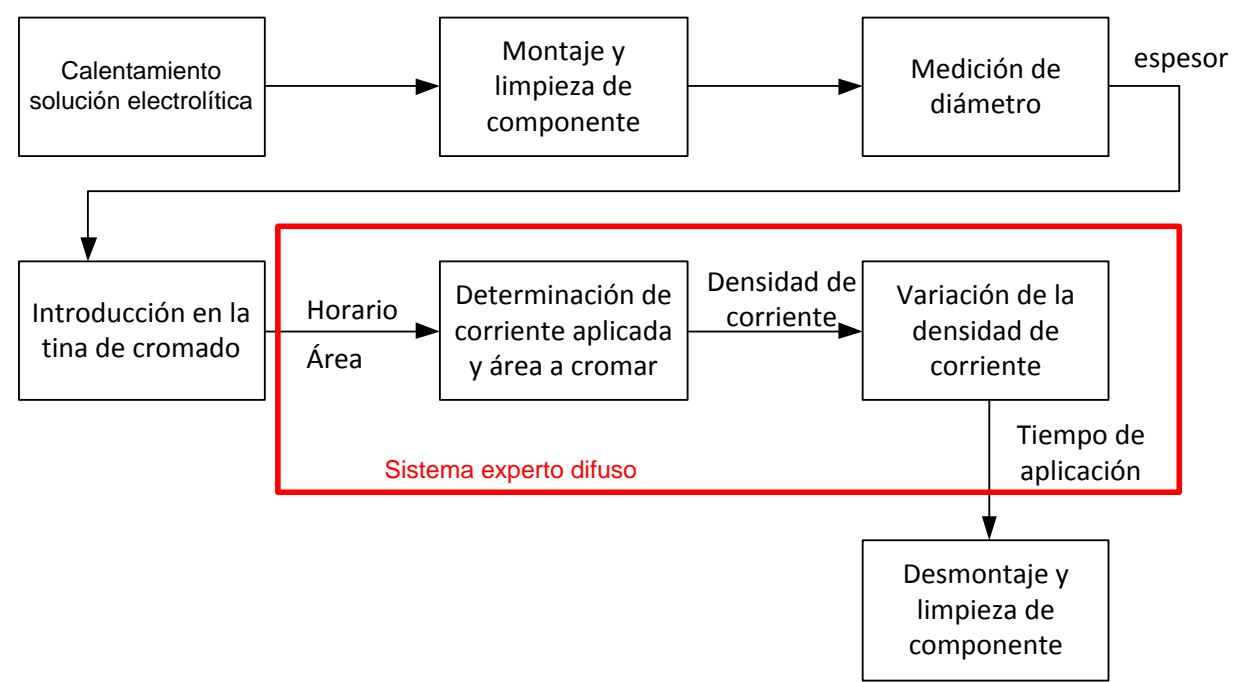

Fig.1: Esquema proceso de cromado.

\section{Lógica Difusa}

La lógica difusa es una teoría matemática que define el grado de pertenencia que tiene un objeto a una clase particular. Este grado de pertenencia es un valor entre cero y uno que indica la adherencia de un grupo a otro (Zadeh, 1965). Los conjuntos difusos son una extensión de los conjuntos clásicos, donde se añade una función de pertenencia, es decir, que a cada elemento del universo se le asocia un grado de pertenencia, definido como un número real entre 0 y 1 (Johansson,1993). Mientras el grado de pertenencia sea más cercano a 1, significa que el elemento pertenece en un mayor grado a dicho conjunto. Caso contrario ocurre si el valor se acerca a 0 . Así es, cómo se introduce el concepto de conjunto o subconjunto difuso y se lo asocia a un determinado valor lingüístico, definido por una palabra o etiqueta lingüística, donde está el nombre del conjunto o subconjunto. Por cada conjunto se define una función de pertenencia o membresía denominada $\mu_{A(x)}$, que indica el grado en que la variable $x$ está incluida en el concepto representado por la etiqueta $\mathrm{A}(0 \leq$ $\left.\mu_{\mathrm{A}(\mathrm{x})} \leq 1\right)$. En particular en este trabajo se utilizan dos tipos de funciones de pertenencia: triangular y trapezoidal. En la Tabla 1, se definen estas dos funciones de pertenencia.

El concepto de lógica difusa define la base de reglas como la manera que tiene un sistema difuso de guardar el conocimiento lingüístico, el cual le permite resolver el problema para el cual ha sido diseñado. Estas reglas son del tipo "Si-Entonces" y asocian uno o más conjuntos difusos de entrada llamados antecedentes con un conjunto difuso de salida llamado consecuente. Para el caso del modelo propuesto, se asocian los conjuntos de entrada con el operador lógico "AND" para tener un conjunto de salida o consecuente (De Negri y De vito, 2006).

Tabla1: Funciones de pertenencia triangular y trapezoidal.

\begin{tabular}{|c|c|c|c|c|c|c|c|}
\hline \multicolumn{4}{|c|}{ Función de pertenencia triangular } & \multicolumn{4}{|c|}{ Función de pertenencia trapezoidal } \\
\hline \multirow{6}{*}{$A(x)=$} & 0 & si & $x \leq a$ & \multirow{6}{*}{$A(x)=$} & 0 & si & $(x \leq a) \circ(x \geq d)$ \\
\hline & $x-a$ & si & $x \in(a, m]$ & & $x-a$ & si & $x \in(a, b]$ \\
\hline & $m-a$ & & & & $b-a$ & & \\
\hline & $b-x$ & si & $x \in(m, b]$ & & 1 & si & $x \in(b, c)$ \\
\hline & $b-m$ & & & & $d-x$ & si & $x \in(h d)$ \\
\hline & 1 & si & $x \geq b$ & & $\overline{d-c}$ & (I & $x \in(0,0)$ \\
\hline \multicolumn{4}{|c|}{$\begin{array}{l}\text { a:límite inferior a; b:límite superior; } m \text { : } \\
\text { valor modal tal que } a<m<b \text {. }\end{array}$} & \multicolumn{4}{|c|}{$\begin{array}{l}\text { a: límite inferior ; d:límite superior ; b: límites de } \\
\text { soporte inferior; c:límite de soporte superior } \mathrm{c} \text {, } \\
\text { tal que } \mathrm{a}<\mathrm{b}<\mathrm{c}<\mathrm{d} \text {. }\end{array}$} \\
\hline
\end{tabular}


Una vez establecida la base de reglas para cada una de las fases del sistema experto difuso, es necesario definir el método de inferencia y agregación que determinan la salida o respuesta del sistema. Mediante el proceso de inferencia, se obtiene el conjunto difuso de salida. Este conjunto es calculado de acuerdo a las reglas establecidas y al método de inferencia utilizado. En este caso, se emplea el método de inferencia por mínimos (Min) de Mamdami definido según la ecuación (1) (Zadeh, 1965).

$$
\min \left(\mu, \mu_{w}(z)\right), \forall z
$$

Donde $\mu_{W}$ es la función de pertenencia del conjunto de salida W.

El método de agregado agrupa todos los conjuntos de salida, de cada una de las reglas establecidas, para obtener un único conjunto difuso. Para el modelo experto difuso propuesto, se utiliza el método de agregación por máximos (Max), descrito por la ecuación (2), el cual agrupa los máximos valores, de cada regla asignada, que han sido obtenidos mediante el proceso de inferencia difuso.

$$
C^{\prime}=\left[\left(z, \mu_{C^{\prime}}(z) / z \in Z\right]\right.
$$

Donde $Z$ es igual al universo del discurso de la variable de salida y $\mu_{C^{\prime}}(z)=\operatorname{Max}\left(C 1^{\prime}, C 2^{\prime}, \ldots, C n^{\prime}\right)$.

El proceso de defusificación consiste en un proceso matemático usado para convertir un conjunto difuso en un número real (Llano et. al., 2007). El conjunto que se ha obtenido del proceso de inferencia y agregación, debe ser transformado en un valor numérico que pueda ser interpretado por elementos externos. Con el fin de determinar qué método de defusificación se emplearía en el modelo, se realizó un análisis previo, utilizando un conjunto menor de datos. El resultado de este análisis arrojó que el método del centroide de área posee un error porcentual más bajo.

En este método, el centro de gravedad es aproximado por el centro de gravedad de un arreglo de masas puntuales, las cuales son el centro de gravedad de cada conjunto de salida correspondiente a cada regla, con masa igual al grado de pertenencia en ese punto de su centro de gravedad. Si se le llama $\delta_{1}$ al centro de gravedad del conjunto difuso de salida $B_{l}$ de la l-ésima regla, el centro de gravedad queda determinado por la ecuación (3).

$$
\mu_{d}=\frac{\sum_{l=1}^{R} \delta_{1} \mu_{B_{l}(\delta l)}}{\sum_{l=1}^{R} \mu_{B_{l}(\delta l)}}
$$

En esta ecuación, $\mathrm{R}$ es el número de reglas. Las reglas que rigen la base de conocimiento corresponden a afirmaciones lógicas que relacionan dos o más objetos e incluyen dos partes, la premisa y la conclusión. Cada una de estas partes consiste en una expresión lógica del tipo "Si- entonces", con una o más afirmaciones objeto-valor conectadas mediante los operadores lógicos 'y', 'o', ó 'no'.

\section{MODELO EXPERTO DIFUSO}

Para la categorización de las variables de entrada y salida, y tal como lo define el concepto de la lógica difusa, es necesario definir cada uno de los parámetros, rangos y funciones de pertenencia que modelarán el proceso real de cromado. Mediante la tabulación del total de datos obtenidos, se determinan los rangos mínimos y máximos de los conjuntos que conforman cada variable seleccionada.

A partir de un análisis gráfico de la agrupación de los datos tabulados, se determinan tanto los rangos de los sub conjuntos difusos, como también las funciones de pertenencia. Cada una de estas funciones debe llevar su respectiva etiqueta lingüística, que cuantifican el valor de dicha función a partir del uso del lenguaje natural o coloquial. La figura 2 presenta un diagrama de flujo del proceso realizado para obtener el modelo difuso de la planta de cromado.

\section{Variables de entrada}

La selección y posterior categorización de las variables involucradas en el proceso de cromado se determina a partir del estudio práctico del proceso y su base teórica. Cuando se definen teóricamente las características que rigen la electrolisis en el baño de cromo, se presentan diversas variables que se involucran y actúan 
entre sí a medida que se realiza el proceso. Al comparar la información obtenida de la teoría con la observación y el análisis de la ejecución práctica del proceso, se identifican las variables más relevantes, destacando aquellas que pueden interferir considerablemente en el resultado final si no se tiene un control sobre ellas durante el proceso. Para tales casos, las variables de entrada a considerar y controlar son: horario, área y espesor

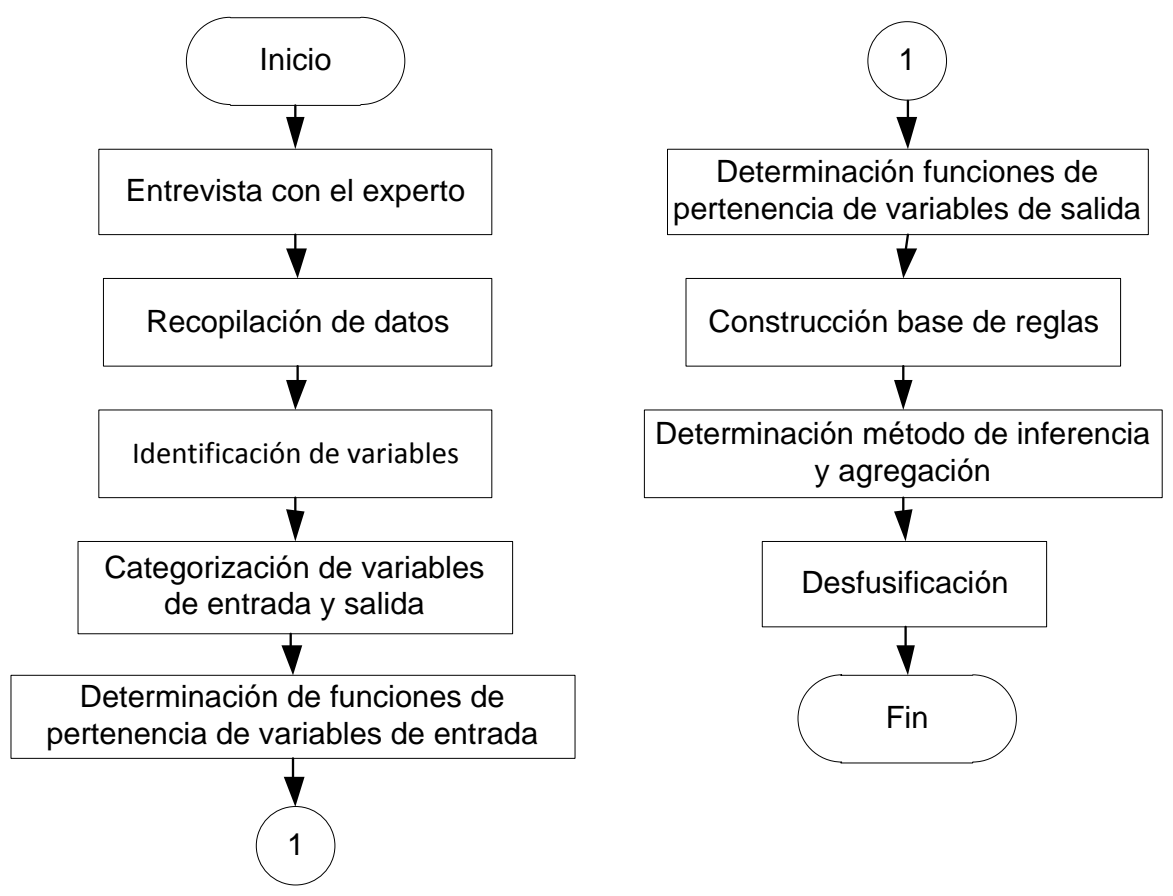

Fig. 2: Diagrama de flujo para obtención del sistema experto difuso

Horario: Representa el período del día (jornada laboral o sobretiempo) donde la pieza ingresa a la tina de cromado. A partir de esta variable el operador determina cuanta densidad de corriente puede aplicar al proceso, teniendo en cuenta si él podrá estar presente cuando el proceso está en ejecución. Se han determinado cuatro subconjuntos para esta variable de entrada (Madrugada, Día, Tarde, Noche), representados con funciones de pertenencia trapezoidal y triangular como se ve en la Figura 3.

El rango para este conjunto va desde las 01:00 [a.m.] hasta las 24:00 [p.m.] que representa la duración de un día. La jornada laboral del operador de la planta comienza a partir de las 08:00 [a.m.] hasta las 18:00 [p.m.]. El periodo de colación es de 12:00 [p.m.] a 13:00 [p.m.], tiempo donde no se introduce ninguna pieza a cromar. Los rangos de cada subconjunto, perteneciente a la variable "Horario" son: Madrugada 01:00-08:00 [a.m.]; Día 06:00 [a.m.] -13:00 [p.m.]; Tarde 12:00-20:00 [p.m.]; Noche 18:00-24:00 [p.m.].

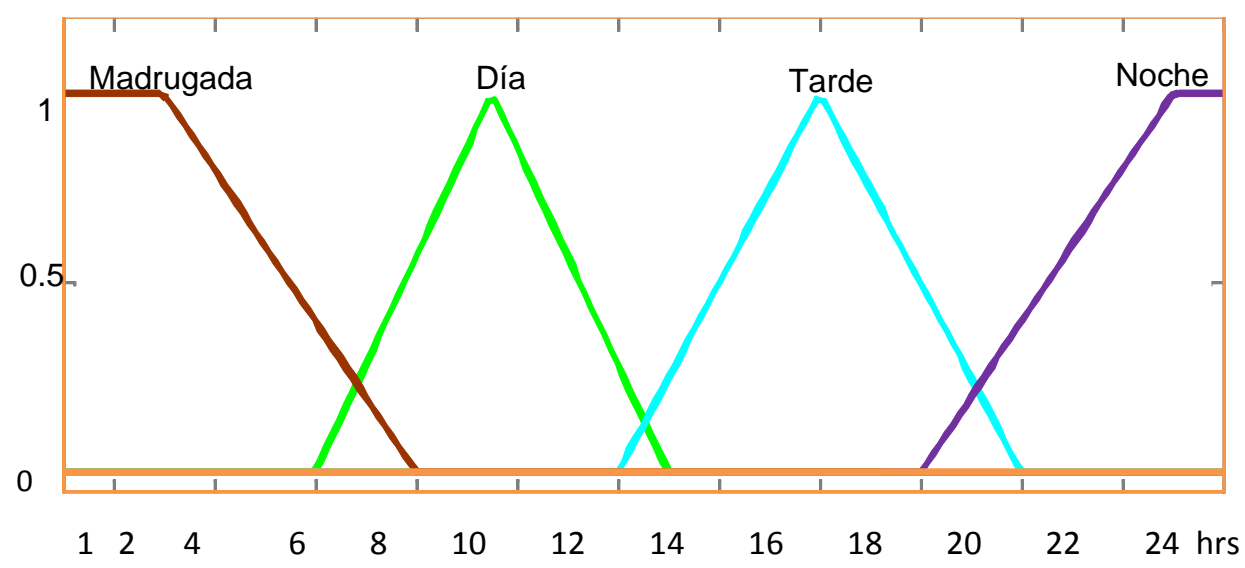

Fig. 3: Función de pertenencia para variable horario. 
Área: Una de las características que condicionan la elección de las variables que rigen la electrólisis son las dimensiones de la pieza a cromar. El área de la superficie que se recubrirá, determina la cantidad de corriente por unidad de área que se aplicará a la solución electrolítica (Densidad de Corriente). El operador de la planta es capaz de realizar variaciones de la corriente a partir de distintos factores. Uno de ellos son las dimensiones de la pieza que se introducirá en la tina. Con estas dimensiones, y mediante la ecuación (4) donde se multiplica el área a cromar por la densidad de corriente, el operador calcula la cantidad de corriente I, que se aplica al iniciar el proceso.

$$
I=A \times D_{C}[a]
$$

En la ec. (4), $A=$ Área $\left[d m^{2}\right] ; D c$ : Densidad de Corriente $\left[\frac{a}{d m^{2}}\right]$

Las funciones de pertenencia empleadas, al igual que en el caso de la variable "Horario", corresponden a funciones trapezoidales y triangulares. Se han establecido tres subconjuntos para definir la variable área. Los rangos de cada subconjunto son: Pequeña 4.5-120 [dm²]; Mediana 40-210 [dm²]; y Grande 130-278 [dm²]. La Figura 4 muestra estas funciones de pertenencia.

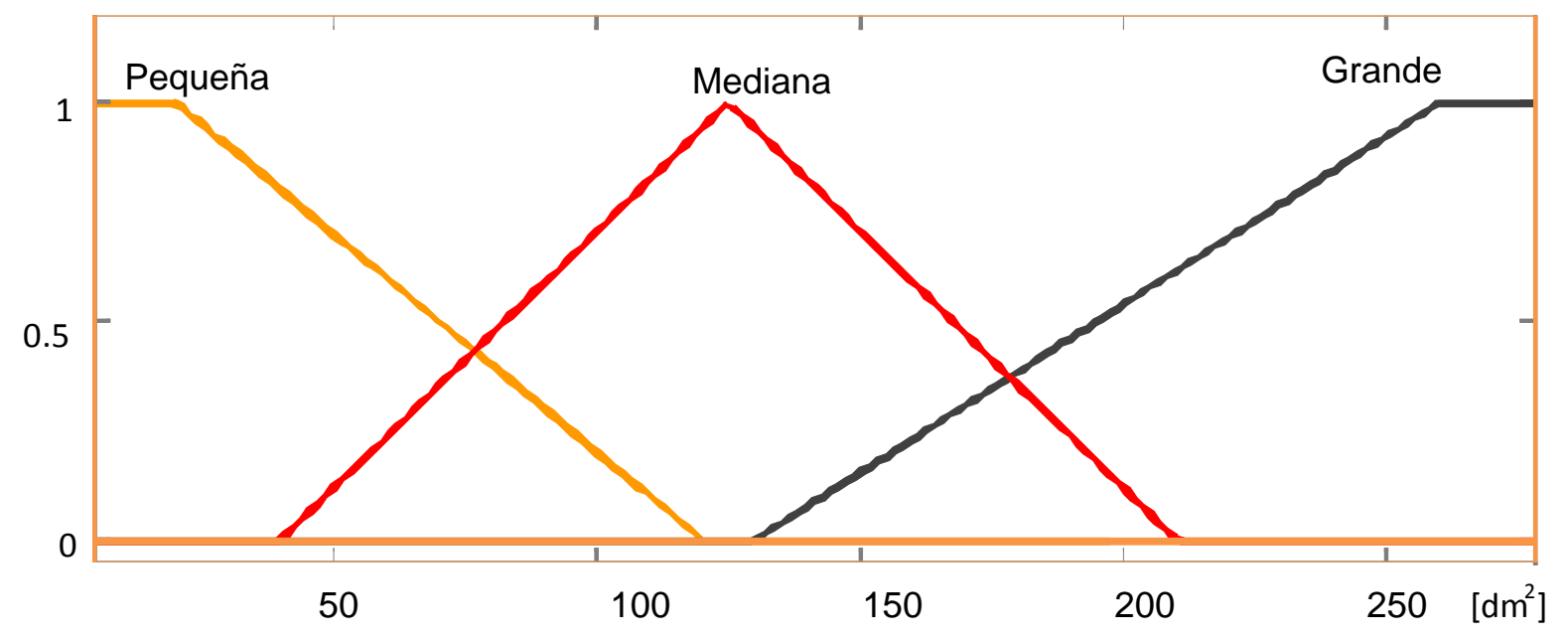

Fig.4: Función de pertenencia para variable área.

Espesor: El espesor también es una de las variables más relevantes del proceso. Dependiendo del espesor de cromo que requiera el componente, será el tiempo que dure el proceso de cromado. Para este caso el operador establece un espesor a partir del diámetro que posee la pieza al llegar a la planta de cromo en comparación con el diámetro nominal establecido en el informe técnico. La diferencia de estos dos diámetros es el espesor de cromo con el que se debe recubrir la pieza, y es dado por:

$$
\Delta \boldsymbol{e}=d_{\text {nominal }}-d_{\text {pieza }}
$$

Donde:

$\Delta e:$ Espesor de cromo a recubrir.

$d_{\text {nominal }}$ : Diámetro nominal de la pieza a cromar.

$d_{\text {pieza }}$ : Diámetro de la pieza previo al cromado.

En los registros que lleva el operador se encuentra el diámetro final de la pieza, después de ser cromada. Esta medida posee un grado de tolerancia con respecto al valor del diámetro nominal del componente, por lo que el resultado no necesariamente debe ser exacto, en comparación con el diámetro nominal. Se estableció un rango de espesores que van desde los $0.05[\mathrm{~mm}]$ a $0.7[\mathrm{~mm}]$, representados por dos funciones de pertenencia trapezoidales y una triangular. Los subconjuntos para esta variable son: Menor (0.05-0.32 [mm]), Regular $(0.2-0.5[\mathrm{~mm}])$ y Mayor $(0.38-0.7[\mathrm{~mm}])$. La Figura 5 muestra la función de pertenencia de la variable espesor. 


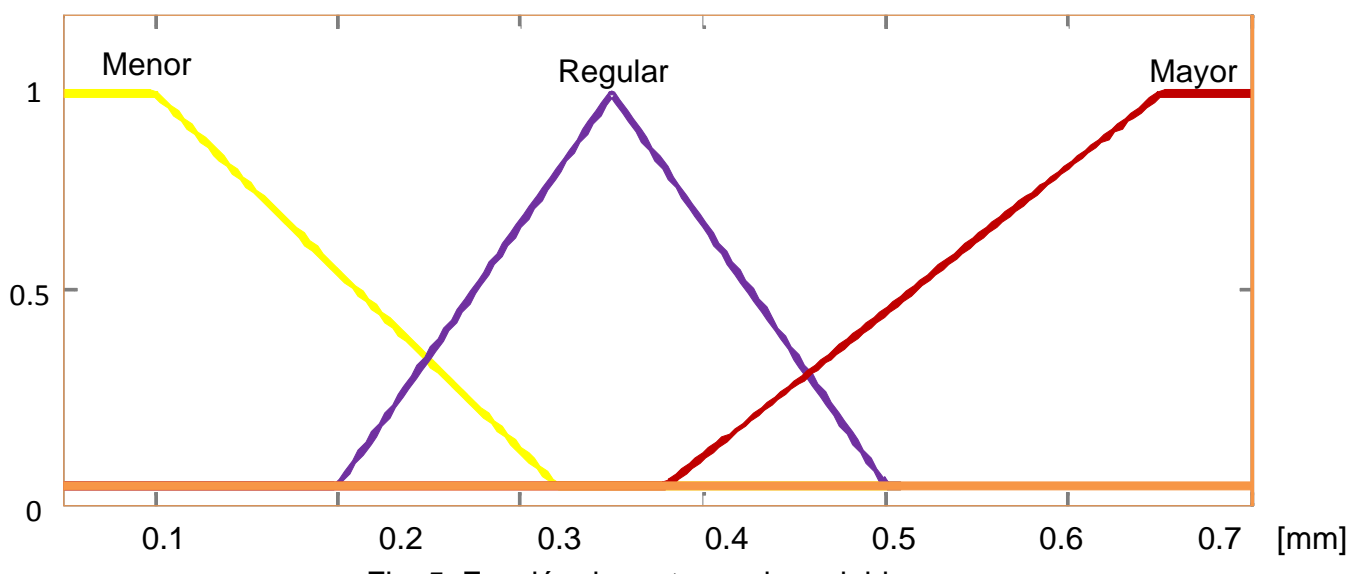

Fig. 5: Función de pertenencia variable espesor.

\section{Variables de salida}

En el proceso real de cromado duro, el operador de la planta es quien toma las decisiones acerca de cada parámetro o variable que se involucra en el proceso. Por esta razón, existe un grado de incertidumbre con respecto a estas decisiones. Según los datos obtenidos del proceso real, el operador puede mantener una densidad de corriente constante durante todo el proceso de cromado, o bien puede variarla según su experiencia. En la mayoría de los casos, el operario hace tres variaciones de la densidad de corriente hasta terminar el proceso. Esta es la razón por la cual se diseña un sistema experto basado en tres fases o etapas, que modelan el proceso real de cromado. En el caso de las variables de salida, se escogen las mismas variables para cada fase del proceso, excepto por los rangos, las funciones de pertenencia y la base de reglas que rige cada modelo. Las variables de salida que se modelan son la densidad de corriente y el tiempo que se aplica esta última durante el proceso.

Densidad de corriente: Cuando se habla de las variables que rigen el cromado duro, se define a la densidad de corriente como el cociente entre la intensidad de corriente y la superficie atravesada por la misma. Dicha variable actúa directamente en el proceso, condicionando la velocidad de deposición del cromo y la calidad de éste. En la ejecución del proceso de cromado, el operario aumenta la densidad de corriente en cada etapa, proceso que involucra a su vez un aumento en la corriente aplicada. Por esta razón es que al modelar esta variable, los rangos que serán implementados en cada fase, son distintos uno del otro. Para cada fase del modelo, se parte de una densidad mínima de 20 [a/dm²], es decir, esta densidad corresponde al límite inferior del rango a usar en cada una de las fases. Para modelar estas dos variables (densidad de corriente con respecto al horario), se analizan todos los valores que puede tomar la densidad de corriente según el horario de entrada a la tina de cromado. De aquí se determina que para el rango de horas entre la 8:00 a.m. y las 11 a.m. es donde se utilizan los valores de densidad de corriente más altos, sobre los 30 [a/dm²]. No obstante, los valores siguen siendo muy variables, razón por la cual se decide utilizar un valor promedio entre todos ellos. Para el resto de las horas de trabajo se realiza el mismo procedimiento, dejando una densidad de corriente promedio y con ello haciendo el proceso menos variable. Las funciones de pertenencia utilizadas para esta variable corresponden a una función triangular y dos trapezoidales. La Figura 6 representa la densidad de corriente de la primera fase del modelo, cuyo rango va desde los 20 [a/dm $\left.{ }^{2}\right]$ a 50 [a/dm²].

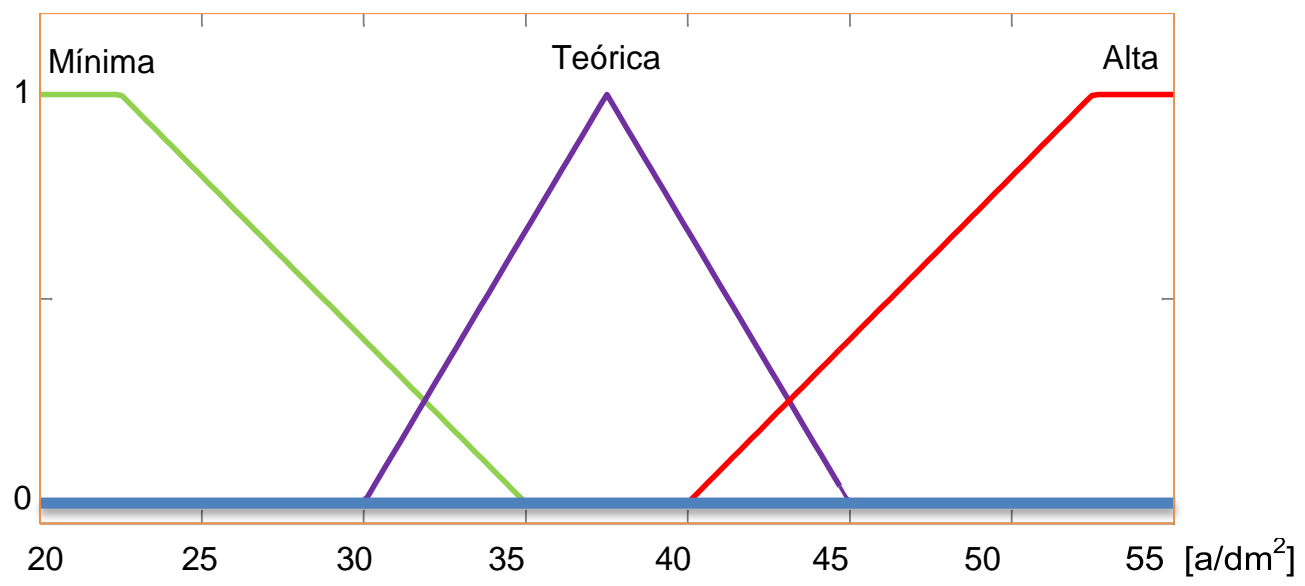

Fig. 6: Función de pertenencia variable densidad de corriente. Fase 1. 
Tiempo de aplicación: Una vez que el operador escoge una densidad de corriente, ésta es aplicada al proceso por un periodo de tiempo que él mismo determina. Factores como el espesor de cromo a recubrir, la velocidad de deposición al inicio del proceso o el periodo del día en el que la pieza ingresa a la tina, influyen en el tiempo que se mantendrá constante dicha densidad de corriente. Por esta razón, este tiempo de aplicación también se considera una variable de salida del sistema. Así para cada una de las fases del proceso, habrá una densidad de corriente constante y un tiempo en que esta densidad se mantendrá.

Para modelar el tiempo de aplicación de la densidad de corriente, se emplean funciones de pertenencia de tipo triangular. Al igual que en caso de la densidad de corriente, los valores lingüísticos que se asignan a los subconjuntos de cada fase, son los mismos. La diferencia que existe entre cada fase son los rangos utilizados, tanto de los conjuntos como de los subconjuntos que conforman las funciones de pertenencia. En la fase uno del proceso, como se ve en la Figura 7, el tiempo de aplicación de la densidad de corriente obtenida, está definida entre un rango que va de 1 a 20 horas.

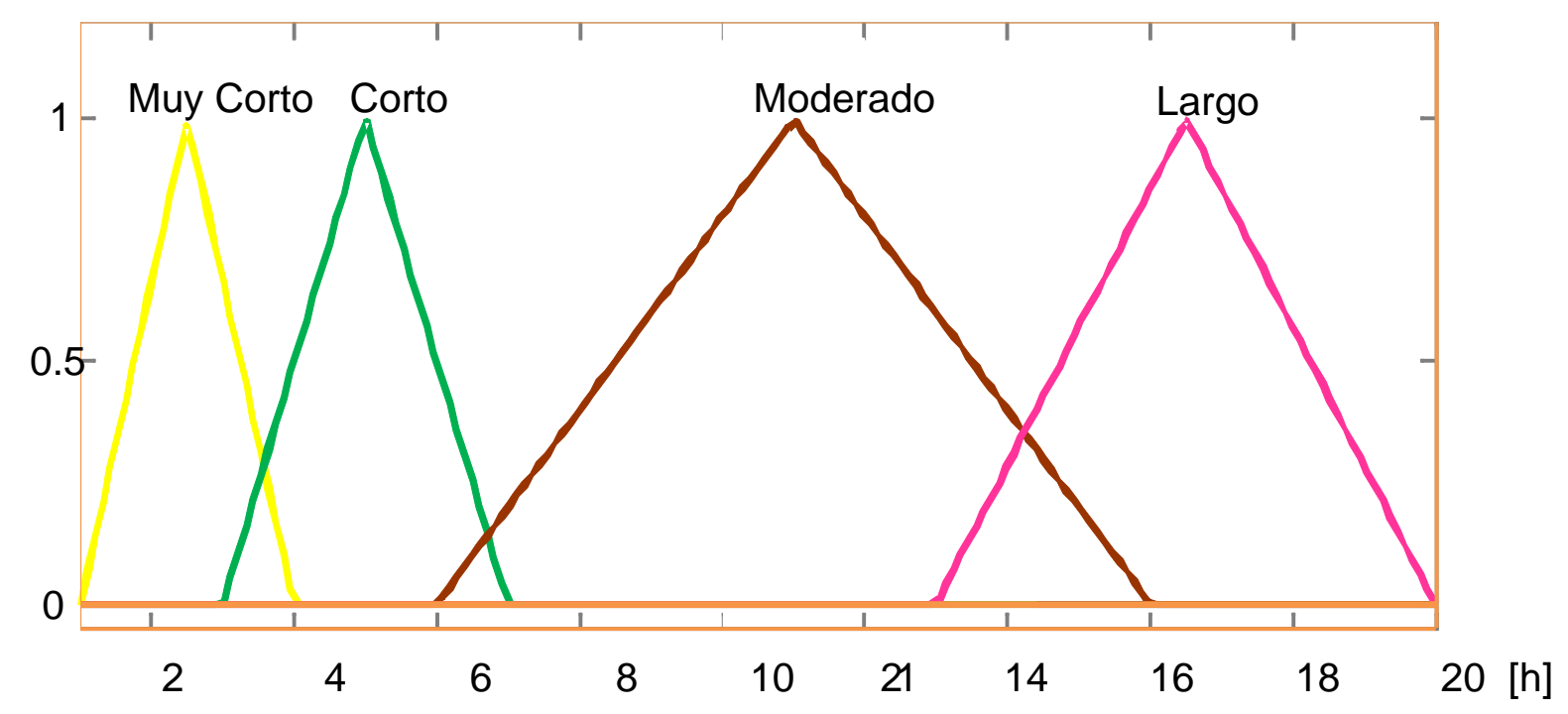

Fig. 7: Función de pertenencia variable tiempo de aplicación. Fase 1.

\section{Base de reglas}

Un conjunto completo de reglas que modelan un problema se obtiene de considerar todas las combinaciones de reglas que es posible establecer teóricamente. Sin embargo, entre todas esas reglas teóricas habrá algunas que no tendrán sentido físico o bien que no se ajustan a las características del problema a resolver. Por esta razón, es que para obtener la base de reglas del modelo propuesto, se analizan todas las posibles combinaciones que ha realizado el operador de la planta. Es decir, mediante los datos tabulados a partir del registro de procesos de cromado realizados, se estudia cada combinación de variables con su respectivo resultado. A partir de ese estudio, se obtiene una base de más de 90 tipos de combinaciones hechas por el experto humano. En cada una de ellas el operador escogió una determinada densidad de corriente, manteniéndola y/o modificándola de acuerdo a las condiciones de entrada del proceso.

Debido a que el modelo planteado está basado en el uso de la lógica difusa, es decir, un sistema que intenta modelar el grado de ambigüedad o incertidumbre que posee el proceso, es necesario reducir la cantidad de combinaciones hechas por el experto humano. Para lograr esta reducción, se utiliza como base de reglas, aquellos casos o combinaciones de variables que han tenido mayor frecuencia a lo largo de los años. Cada fase del modelo planteado es representado por una base de reglas distinta. La estructura de estas reglas difiere según el tipo de reglas que se utilice. Para este caso se utilizan las reglas del tipo Mamdani, que permiten asociar a cada variable de entrada un conjunto de salida, que posteriormente será la respuesta del sistema (acciones de control). Además, este tipo de reglas posee la ventaja de ser adaptable en caso de una incorporación de más conocimientos y/o experiencia. Para el caso de la fase 1 del modelo planteado, se proponen una base de 17 reglas. A cada una de ellas se le asigna un valor que está entre 0 y 1 . Este valor determina el peso de dicha regla. Esto es importante cuando una regla tiene más o menos fuerza que otras de la base de reglas. En la tabla 2, se muestran algunas reglas correspondientes a la fase uno, a modo de ejemplo. En la segunda fase y la última fase del modelo, la base de reglas está conformada por 16 reglas y 17 reglas difusas respectivamente. 
Tabla 2: Ejemplo base de reglas fase 1

\begin{tabular}{|l|l|}
\hline 1.- & $\begin{array}{l}\text { Si Horario es Madrugada and Área es Pequeña and Espesor es Menor entonces Densidad de Corriente } \\
\text { es Teórica and Tiempo de Aplicación es Corto. }\end{array}$ \\
\hline 2.- & $\begin{array}{l}\text { Si Horario es Madrugada and Área es Grande and Espesor es Menor entonces Densidad de Corriente } \\
\text { es Mínima and Tiempo de Aplicación es Moderado. }\end{array}$ \\
\hline 3.- & $\begin{array}{l}\text { Si Horario es Día and Área es Pequeña and Espesor es Menor entonces Densidad de Corriente es Alta } \\
\text { and Tiempo de Aplicación es Muy Corto. }\end{array}$ \\
\hline 4.- & $\begin{array}{l}\text { Si Horario es Día and Área es Pequeña and Espesor es Regular entonces Densidad de Corriente es } \\
\text { Mínima and Tiempo de Aplicación es Muy Corto. }\end{array}$ \\
\hline 5.- & $\begin{array}{l}\text { Si Horario es Día and Área es Mediana and Espesor es Menor entonces Densidad de Corriente es } \\
\text { Mínima and Tiempo de Aplicación es Muy Corto. }\end{array}$ \\
\hline 6.- & $\begin{array}{l}\text { Si Horario es Día and Área es Pequeña and Espesor es Menor entonces Densidad de Corriente es } \\
\text { Mínima and Tiempo de Aplicación es Muy Corto. }\end{array}$ \\
\hline 7.- & $\begin{array}{l}\text { Si Horario es Día and Área es Pequeña and Mayor es Regular entonces Densidad de Corriente es Mínima } \\
\text { and Tiempo de Aplicación es Muy Corto. }\end{array}$ \\
\hline 8.- & $\begin{array}{l}\text { Si Horario es Tarde and Área es Mediana and Espesor es Menor entonces Densidad de Corriente es } \\
\text { Mínima and Tiempo de Aplicación es Corto. }\end{array}$ \\
\hline
\end{tabular}

\section{RESULTADOS Y DISCUSIÓN}

Se presenta una selección de los resultados obtenidos en este trabajo. Los resultados mostrados en la Figura 8 , corresponden a la primera fase del proceso de cromado duro. Como se ha mencionado anteriormente, es en esta fase donde se analizan todas las condiciones de entrada previas a la ejecución del proceso de cromado. Es decir, es necesario conocer el estado actual del componente que será cromado (acabado superficial, dimensiones de la pieza, espesor de cromo), como también las condiciones operacionales donde es ejecutado el proceso (Horario de trabajo). Cada una de estas condiciones de entrada, son ingresadas al modelo experto difuso, el cual simula la toma de decisiones que realiza el operador de la planta. La Figura 8 entrega los resultados obtenidos al modelar la densidad de corriente con respecto al horario, comparando dichos resultados con los datos reales del proceso.

Otra de las variables modeladas es el tiempo de aplicación de la densidad de corriente. La Figura 9 muestra los resultados obtenidos al modelar el tiempo de aplicación de la densidad de corriente con respecto al horario y comparar dichos resultados con los datos reales del proceso. En la gráfica se aprecia que la mayor diferencia entre el modelo propuesto y el proceso real se encuentra en horarios entre las 11:00 a.m. y las 18:00 p.m. Esta diferencia debe a que en el registro de los datos del proceso, existen casos puntuales donde los tiempos de cromado son muy extensos (40 [h]). Por ello las fases donde se varía la densidad de corriente tienen un tiempo de aplicación mayor, lo que hace que existan grandes diferencias entre los tiempos de aplicación de la densidad de corriente.

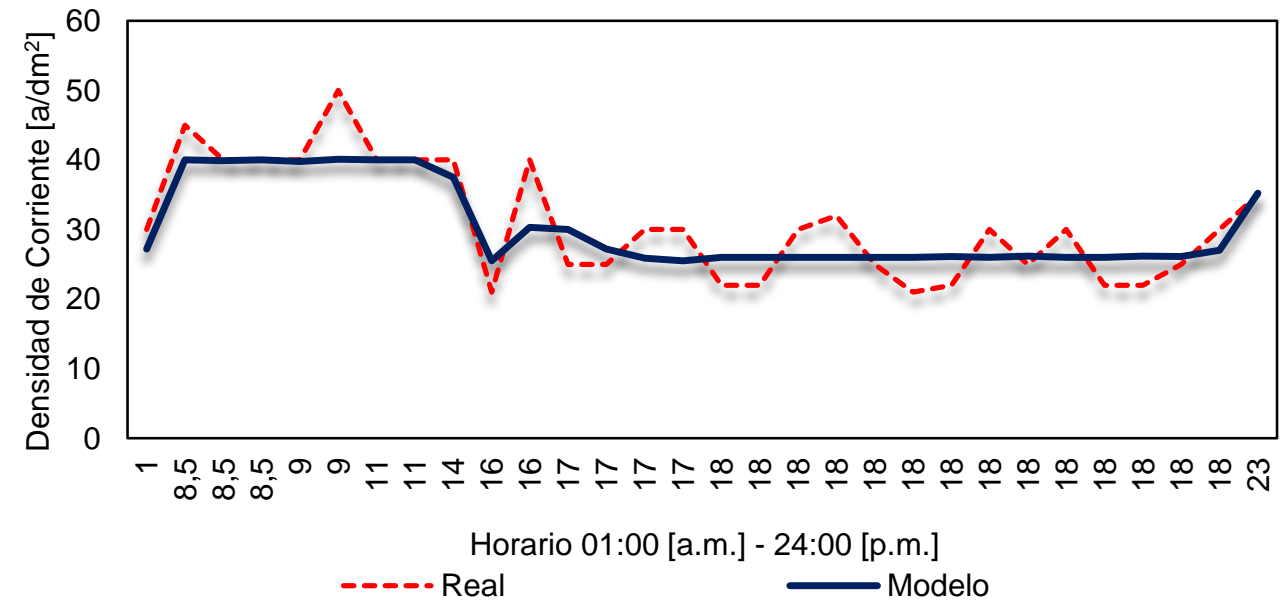

Fig. 8: Densidad de corriente Real y el Modelo versus el horario. Fase 1 del proceso. 


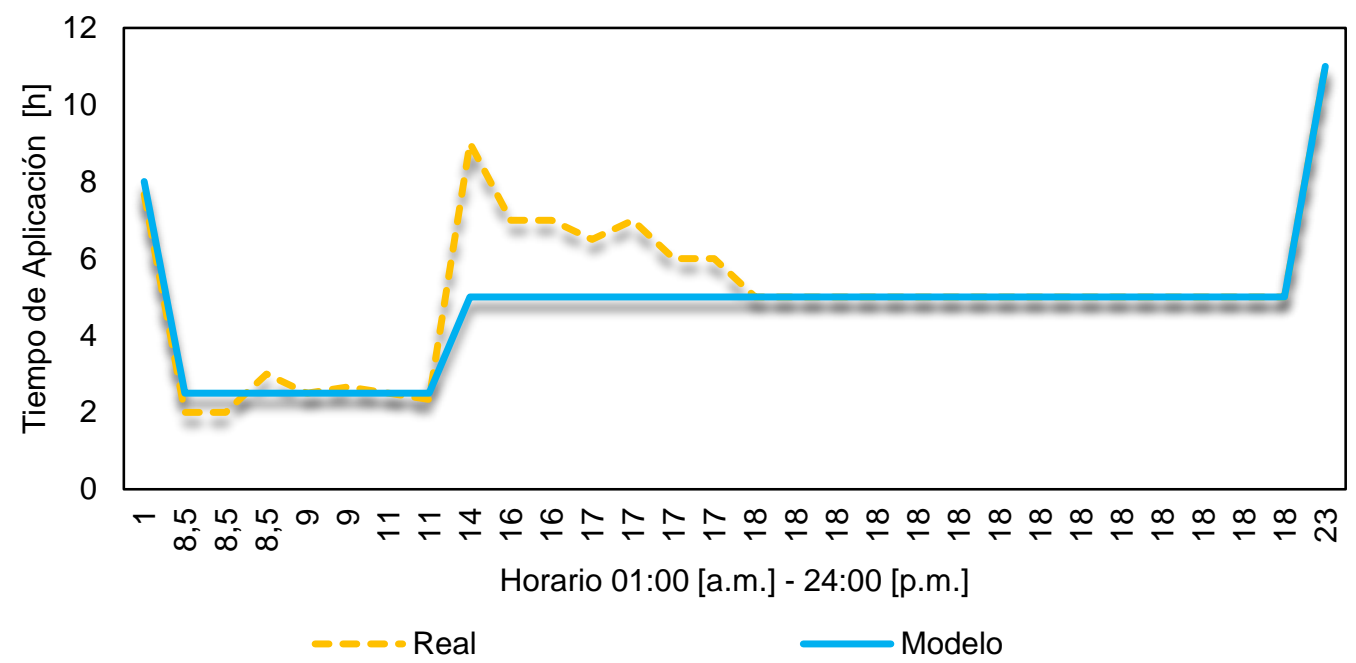

Fig. 9: Tiempo de aplicación de la densidad de corriente Real /Modelo versus el horario. Fase 1 del proceso.

Al igual que con la variable "Horario", también se realiza la comparación de la variable densidad de corriente vs el área obtenida a través del modelo y los valores reales. Lo mismo se presenta para la variable tiempo de aplicación vs. área. En las Figuras 10 y 11 se observa dicho comportamiento, donde la línea de tendencia del modelo y la del proceso real siguen el mismo patrón, lo que significa que el modelo propuesto es representativo del proceso real de cromado.

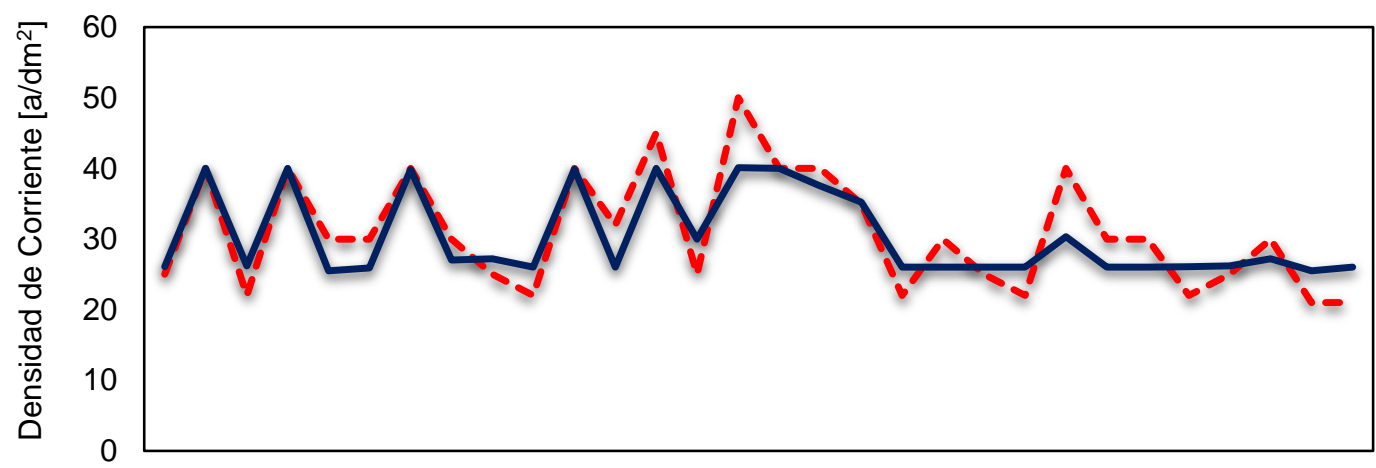

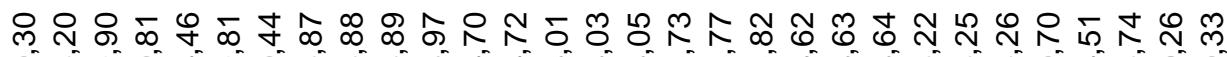

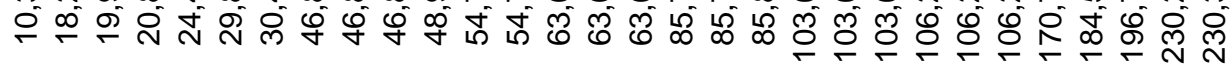

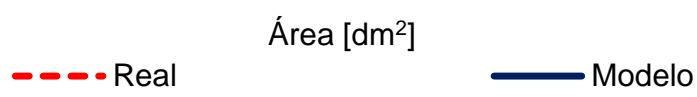

Fig.10: Densidad de corriente Real/Modelo versus el área. Fase 1 del proceso

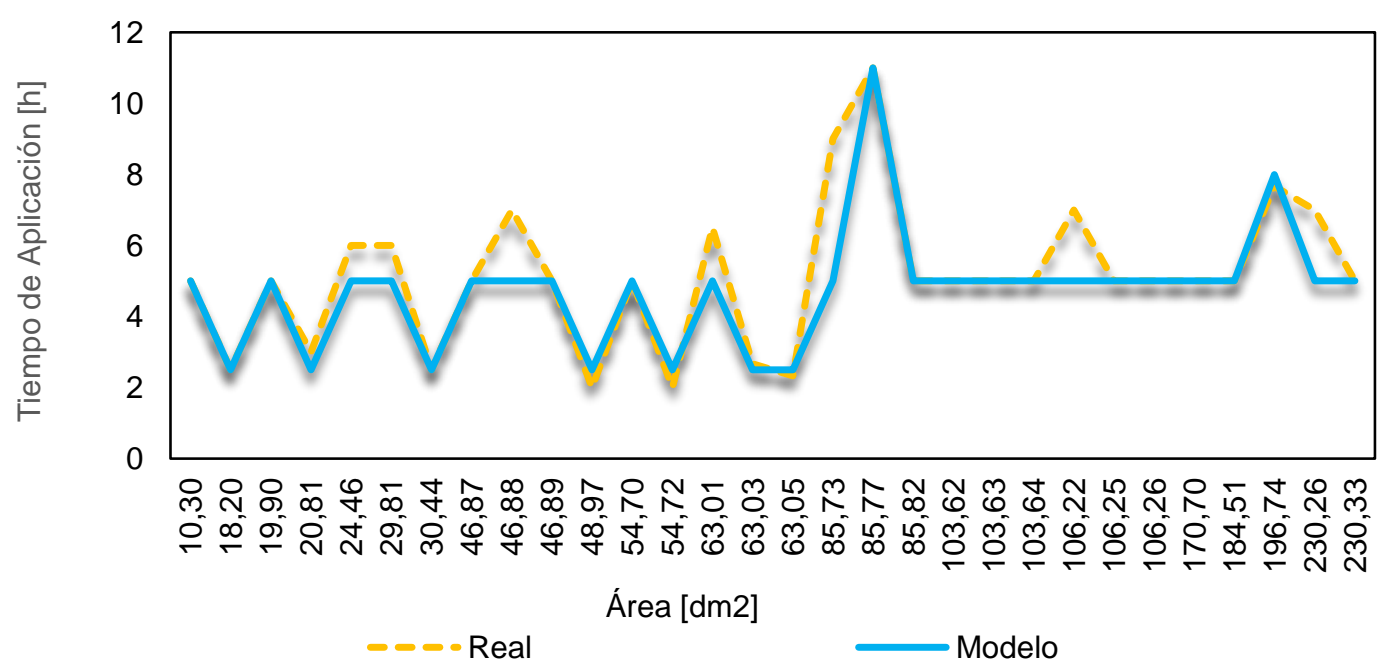

Fig.11: Tiempo de aplicación de la densidad de corriente Real /Modelo versus el área. Fase 1 del proceso. 
La fase 3 corresponde a la última etapa del sistema experto difuso, aunque de acuerdo a los registros del proceso de cromado, la cantidad de veces que el operador varía la densidad de corriente supera las tres veces. Esto implicaría tener un mayor número de fases. Tal como se ha descrito anteriormente, el modelo difuso propuesto fija el proceso de cromado en tres fases, siendo esta última, la etapa que finaliza el proceso.

Las condiciones de entrada de esta fase corresponden a los resultados que se obtuvieron de la segunda fase del proceso. En esta fase se obtuvieron los mejores resultados, en comparación con las otras dos fases del proceso. Sólo se produjo un $8,2 \%$ de error al modelar la densidad de corriente, comparándola con los resultados del proceso real de cromado. Las Figuras 12 y 13 muestran el comportamiento de la densidad de corriente y su tiempo de aplicación con respecto al área de la superficie a cromar. En el caso de la densidad de corriente, la línea de tendencia tiene una leve tendencia al descenso a medida que aumenta el área de cromado. No obstante, presenta algunos puntos donde, para un área similar, la densidad de corriente es muy variante. Un ejemplo de ello es el caso donde el rango del área a cromar está entre los $48-63$ [dm²].

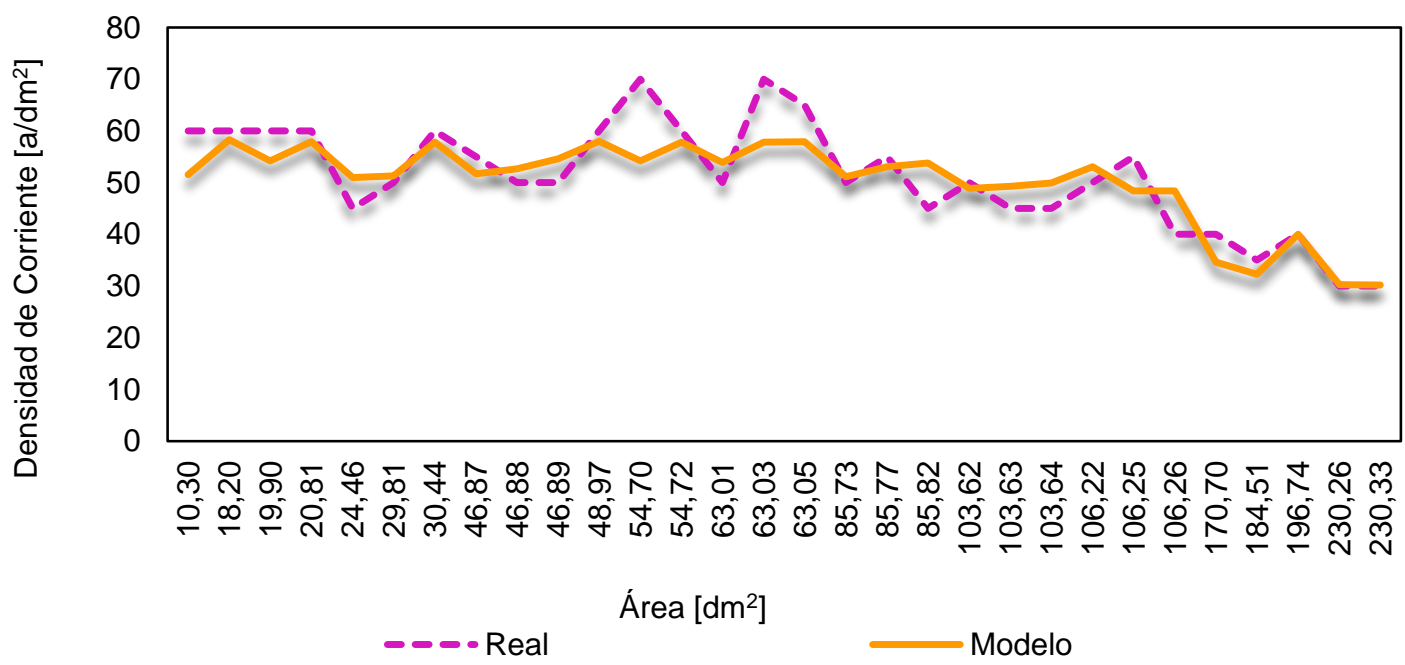

Fig.12: Densidad de corriente Real/Modelo versus el área. Fase 3 del proceso.

En cuanto al tiempo de aplicación de la densidad de corriente, éste presenta un comportamiento estable a medida que aumenta el área de cromado. Las variaciones que presenta esta variable se deben a que el tiempo de aplicación de la densidad de corriente depende de los horarios donde se está trabajando.

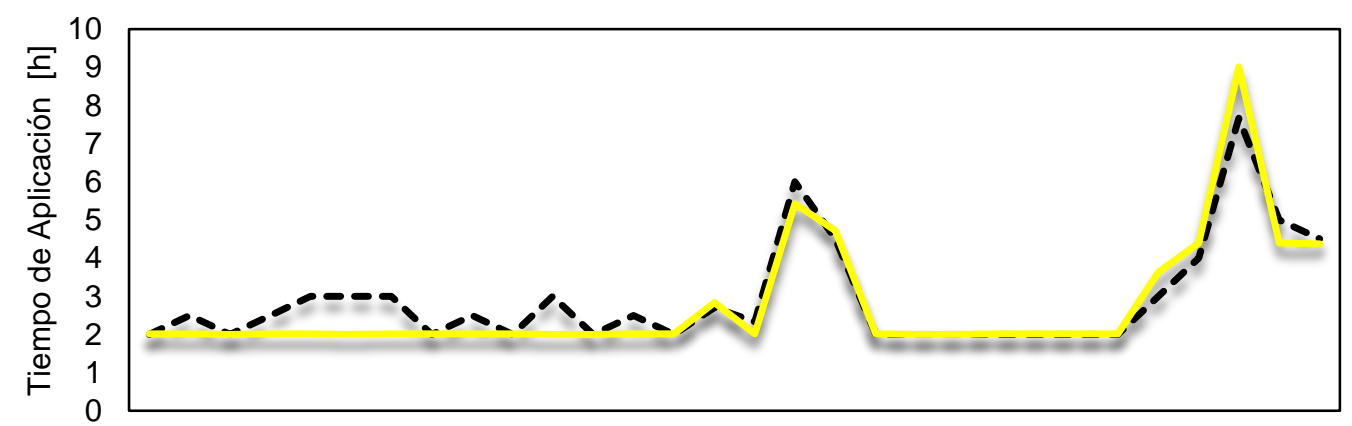

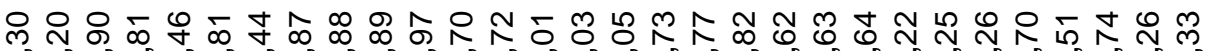
ำ

$$
\text { Área }\left[\mathrm{dm}^{2}\right]
$$

Fig.13: Tiempo de aplicación de la densidad de corriente Real/Modelo versus el área. Fase 3 del proceso.

Tal vez la variable más relevante en el proceso de cromado sea el espesor de éste. Las Figuras 14 y 15 muestran el comportamiento de la densidad de corriente y su tiempo de aplicación con respecto al espesor de cromo que se aplica al componente. Ambas gráficas muestran una línea de tendencia que presenta muchas variaciones a medida que aumenta el espesor de cromo. En esta etapa la cantidad de densidad de corriente que se aplica depende de las condiciones en las que se encuentra la pieza. El operador realiza una evaluación del proceso y toma decisiones sobre el mismo, pasando de la fase dos a la fase tres, o última etapa del proceso. Esto explica el por qué existe tanta variación en cuanto a los valores que puede tomar la 
densidad de corriente a partir de las variables de entrada del proceso. En cada una de las gráficas, obtenidas para las tres fases del proceso, se aprecia que tanto la curva del proceso real de cromado como la del modelo experto difuso, siguen un comportamiento similar, siendo menos variante la línea de tendencia de modelo difuso. Son pocos los casos en donde existe un error que supera el $15 \%$, entre el proceso real y el modelo propuesto. Por esta razón se puede decir que el sistema experto difuso cumple satisfactoriamente con su objetivo y es representativo del proceso real de cromado duro.

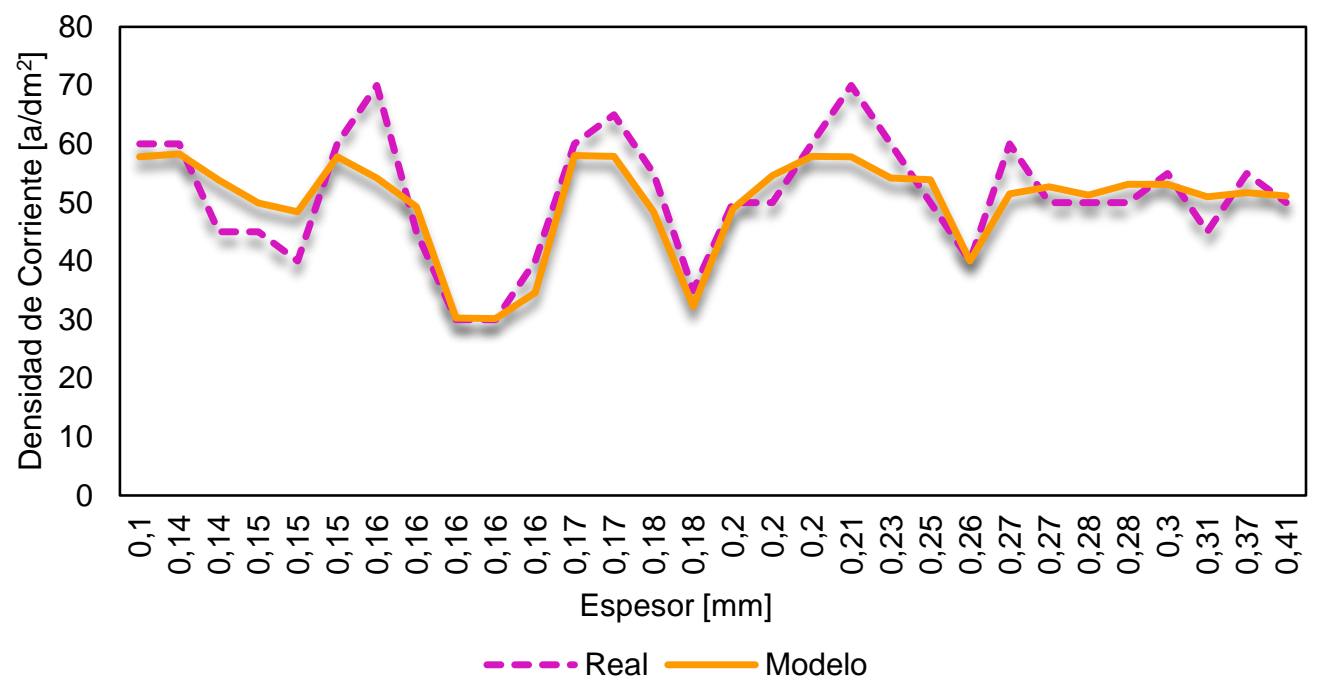

Fig.14: Gráfico comparativo entre la densidad de corriente Real/Modelo versus el espesor, para la fase 3 del proceso.

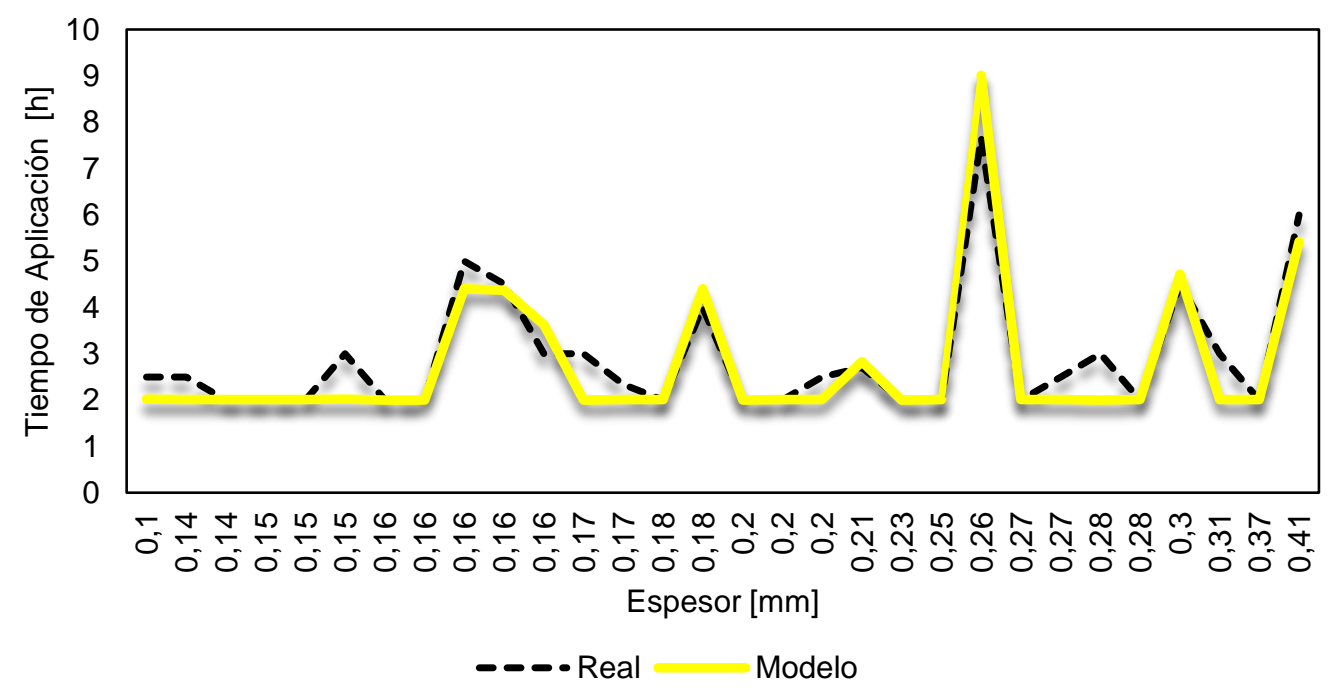

Fig.15: Gráfico comparativo entre el tiempo de aplicación de la densidad de corriente Real/Modelo versus el espesor, para la fase 3 del proceso.

\section{CONCLUSIONES}

Los resultados obtenidos muestran que:

1) El sistema experto difuso encontrado representa adecuadamente el proceso real de cromado duro. 2) La modelación del proceso permite ajustar el número de etapas del proceso. En la ejecución del proceso de cromado existe una variación de la densidad de corriente que divide el proceso en distintas etapas. Con el uso del sistema experto se logra estandarizar el proceso de cromado duro, ajustándolo a tres etapas en la cuales se varía la densidad de corriente según las condiciones de entrada del proceso. 3) La utilización del concepto de lógica difusa, las reglas difusas, base de reglas y los operadores de Mamdami (Min-Max), ha permitido representar el conocimiento aportado por el operador de la planta, con todo el grado de incertidumbre o ambigüedad que existe ante cada decisión que él toma sobre el proceso. 4) La utilización del sistema experto difuso permitirá reducir los costos, especialmente los pagos de horas extras que realiza el operador de la planta de cromado. 5) La automatización del proceso de cromado permitirá al operador estar menos expuesto a los gases nocivos que se desprenden de la solución electrolítica cuando se ejecuta el proceso de cromado. 


\section{AGRADECIMIENTOS}

Se agradece el apoyo a la Dirección de investigación de la Universidad de la Serena, a través del proyecto DIDULS PR17351.

\section{REFERENCIAS}

Aydin Z., G. Aldic y H. Cimenoglu, An Investigation on the Mechanical properties of the Hard Chromium layer Deposited by Brush Plating Process on AISI H13 Steel, Archives of Materials Science an Engineering, 65(2), 87-92 (2014)

D’ Negri, C. y E. De Vito, Introducción al razonamiento aproximado: Lógica Difusa, Revista Argentina de Medicina Respiratoria, Instituto de Investigaciones Médicas Alfredo Lanari, U. de Buenos Aires, Argentina, 4, 126 - 136 (2006)

Druesne, F., M. Afzali, Electroplating Simulation and Design Tool, Proceeding of the Institution of Mechanical Engineers, Part B, Journal of Engineering manufacture, 217(5), 705-707 (2003)

Johansson, R., System modeling and identification, Information and Systems Science series, Prentice-Hall, New Jersey (1993)

Kullawong, T., S. Butdee, Integrating Reliability-Centered Maintenance with Cost Optimization \& Application in Plant of Hard Chrome Plating, International Journal of Industrial Engineering and Management (IJIEM), 6 (2), 85-92 (2015)

Lyphout, C., S. Björklund y otros cuatro autores, Screening Design of Supersonic Air Fuel Processing for hard metal Coatings, Journal of Thermal Spray Technology, 23(8), 1323-1332 (2014)

Llano L., G. Zapata, D. Ovalle, Sistema de Inferencia Difuso para Identificar Eventos de Falla en Tiempo Real del STE usando Registros SOE, Revista Avances en Sistemas e Informática, Universidad Nacional de Colombia, Medellín, 4(2), 125-134 (2007)

Llata, J.R. y otros cuatro autores, Aplicación de Inteligencia Artificial en Sistemas Automatizados de Producción, Revista Iberoamericana de Inteligencia Artificial, 4 Valencia, España, 10, 100-110 (2000)

Mayacela, C., N. Otañez, Diseño, Construcción e Implementación de un Sistema de Pulido Electrolítico de Materiales ferrosos para el Proceso de Cromado en la Empresa Ingenieros Lara Nuñez Cia Ltda. (2013)

Obaid, N., R. Sivakumaran, J. Lui y A. Okunade, Modelling the Electroplating of Hexavalent Chromium, COMSOL Conference, October (2013)

Pam, S. Y., M. McCormick, D. How De y A. Naismith, The Modelling of Electroplating Baths and its Application to Jig Design, The International Journal of Surface Engineering and Coating, 59(1), 61-67 (1981)

Pino, R., A. Gómez, N. de Abajo, Introducción a la Ingeniería Artificial: Sistemas Expertos, Redes Neuronales Artificiales y Computación Evolutiva, Editorial Servicios de Publicaciones Universidad de Oviedo, 89-145, Oviedo, España (2001)

Qian, S., D. Zhu y otros tres autores, Generating Micro-Dimples array on the hard chrome-Coated Surface by Modified Through mask Electrochemical Micromachining, International Journal of Advanced Manufacturing Technology, 47, 11211127 (2010)

Ruiz, J., C. Parra y otros tres autores, Modelamiento del Proceso de Recubrimiento de Instrumental Quirúrgico con Cromoduro, Revista Facultad de Ingeniería, 30, 89-94 (2003)

Sánchez, F., J.A. Vilán, P.J. García y J. Del Coz, The Use of Design of Experiments to Improve a Neural Network Model in Order to Predict the Thickness of the Chromium Layer in a Hard Chromium Plating Process, Mathematical and Computer Modelling, 52 (7-8), 1169-1176 (2010)

Sánchez, F., P.J. García, J. Del Cos, J.A. Vilán, Evolutionary Support Vector Regression Algorithm Applied to the Prediction of the Thickness of the Chromium Layer in a Hard Chromium Plating Process, Applied Mathematics and Computation, 227, 164-170 (2014)

Telukdarie, A., Development of a Hybrid Fuzzy-Mathematical Cleaner Production Evaluation Tool for Surface Finishing, Tesis Doctorado de Tecnología, Durban University of Technology, Sudafrica (2007)

Vargas, V., Sistema de Visión Artificial para el Control de Calidad en Piezas Cromada. Tesis Maestría en Ingeniería de Manufactura, Instituto Politécnico Nacional, SEPI, ESIME, Unidad Azcapotzalco, México (2010)

Vaschetti, J., F. Magnago y V. Sauchelli, Control Automático de Voltaje en Sistemas Eléctricos de Potencia Basado en Sistemas Expertos, Información Tecnológica, 23(5), 69-84 (2012)

Vinokurov, E.G., Meshalkin, V.P. y otros cuatro autores, System Analysis of the Efficiency and Competitiveness Of Chroming Technologies, Theoretical Foundations of Chemical Engineering, 50 (5), 730-738 (2016)

Zadeh, L., Fuzzy Sets, Information and Control, 8, 338-353 (1965)

Zhu D., N.S. Qu y otros cuatro autores, Electrochemical Micromachining of Microstructures of Micro Hole and Dimple Array, CIRP Annals- Manufacturing Technology, 58, 177-180 (2009) 